C. Hai Lim

Nagoya Math. J.

Vol. 125 (1992), 73-92

\title{
THE JACOBIAN OF A CYCLIC QUOTIENT OF A FERMAT CURVE
}

\author{
CHONG HAI LIM
}

\section{§ 0. Introduction}

Fix a positive integer $m$. Let $F_{m}$ denote the Fermat curve over $\mathbf{Q}$ of degree $m$, given by the projective equation

$$
X^{m}+Y^{m}+Z^{m}=0 .
$$

Let $\mu_{m} \subseteq \overline{\mathbf{Q}}$ be the group of $m$-th roots of unity, $\Delta$ be the image of $\mu_{m}$ in $\mu_{m}^{3}$ under the diagonal embedding, and let $G_{m}=\mu_{m}^{3} / \Delta$. Then $G_{m}$ acts on $F_{m}$ :as follows:

$$
\left(\xi_{1}, \xi_{2}, \xi_{3}\right) \bmod \Delta:(X, Y, Z) \longrightarrow\left(\xi_{1} X, \xi_{2} Y, \xi_{3} Z\right) .
$$

The group ring $\mathbf{Z}\left[G_{m}\right]$ acts on the Jacobian $J_{m}$ of $F_{m}$. Let $K=\mathbf{Q}\left(\mu_{m}\right)$. Then $J_{m} / K$ has CM by $\mathbf{Z}\left[G_{m}\right]$ [4].

Let $a, b, c \in \mathbf{Z}$, with $a+b+c=0,(a, b, c, m)=1$, and none of $a, b, c$ divisible by $m$. Let $\Gamma_{a, b, c}^{m}$ be the following subgroup of $G_{m}$ :

$$
\left\{\left(\xi_{1}, \xi_{2}, \xi_{3}\right) \in \mu_{m}^{3} \mid \xi_{1}^{a} \xi_{2}^{b} \xi_{3}^{c}=1\right\} / \Delta .
$$

Then the quotient curve

$$
F_{a, b, c}^{m}=\Gamma_{a, b, c}^{m} \backslash F_{m}
$$

is defined over $\mathbf{Q}$, and has equation $y^{m}=(-1)^{c} x^{a}(1-x)^{b}$. Its Jacobian $J_{a, b, c}^{m}$ has CM by

$$
\mathrm{Z}\left[G_{m} / \Gamma_{a, b, c}^{m}\right]
$$

Let $g$ be a generator of the cyclic group $G_{m} / \Gamma_{a, b, c}^{m}$, and let $f_{m}(x)$ denote the $m$-th cyclotomic polynomial. Then the sum of the images of the maps

$$
J_{a, b, c}^{d} \longrightarrow J_{a, b, c}^{m}
$$

induced from $F_{a, b, c}^{m} \rightarrow F_{a, b, c}^{d},(x, y) \rightarrow\left(x, y^{m / d}\right)$, as $d$ varies over the set of

Received January 7, 1991. 
proper divisors of $m$, generates the abelian subvariety $f_{m}(g) J_{a, b, c}^{m}$ of $J_{a, b, c}^{m}$. We define $\left(J_{a, b, c}^{m}\right)^{\text {new }}$ to be the quotient of $J_{a, b, c}^{m}$ by $f_{m}(g) J_{a, b, c}^{m}$.

In [8], Koblitz-Rohrlich determined the necessary and sufficient conditions for $\left(J_{a, b, c}^{m}\right)^{\text {new }}$ to be non-simple and its decomposition into simple factors up to isogeny in the case when $(m, 6)=1$. Aoki [1] has solved this problem for all sufficiently large $m$. In $\S 2$, we use the above mentioned results to determine the ring of rational endomorphisms of some non-simple $\left(J_{a, b, c}^{m}\right)^{\text {new }}$.

In the rest of this paper, we let $p$ be an odd prime, fix a cyclic quotient curve of $F_{p}$ and denote its Jacobian by $A$. From the work of Koblitz-Rohrlich [8] and Schmidt [12], we know that $A$ is either absolutely simple or isogeneous to a cube of an absolutely simple abelian variety over the $p$-th cyclotomic field $\mathbf{Q}\left(\mu_{p}\right)$. When $A$ is simple, End $(A)$ is isomorphic to the ring of integers in $\mathbf{Q}\left(\mu_{p}\right)$. In $\S 4$, we shall completely characterize the endomorphism ring of $A$ whenever it is non-simple. We then use this information to show in $\S 6$ that $A$ is in fact isomorphic over $\mathbf{Q}\left(\mu_{p}\right)$ to a cube of a simple abelian variety. A special case of this result $(p=7)$ is that the Jacobian $\operatorname{Jac}(C)$ of the Klein curve

$$
C: X^{3} Y+Y^{3} Z+Z^{3} X=0
$$

is isomorphic to a cube of an elliptic curve [10] (in fact, the elliptic modular curve $\left.J_{0}(49)\right)$.

\section{$\S 1$. Preliminaries}

For the Fermat curve $F_{m}$, let $x=X / Z$ and $y=Y / Z$. Now let $r, s$, $t \in \mathbf{Z}, 0<r, s, t<m$ and $r+s+t \equiv 0(\bmod m)$. Then

$$
w_{r, s, t}=x^{r-1} y^{s-1} \frac{d x}{y^{m-1}}
$$

is a differential form of the second kind on $F_{m}$. $G_{m}$ is generated by $\sigma=(\zeta, 1,1)$ and $\tau=(1, \zeta, 1)$, where $\zeta$ is a fixed primitive $m$-th root of unity, and the forms $w_{r, s, t}$ are eigenforms for the action of $G_{m}:\left(\sigma^{j} \tau^{k}\right)^{*} w_{r, s, t}=$ $\zeta^{r j+s k} w_{r, s, t}$. Since the characters on $(\mathbf{Z} / m \mathbf{Z})^{2}$ are mutually distinct,

$$
\Omega=\left\{w_{r, s, t} \mid 0<r, s, t<m, r+s+t \equiv 0(\bmod m)\right\}
$$

is a basis of the de Rham cohomology $H_{\mathrm{DR}}^{1}\left(F_{m}\right) . \quad \Omega_{1}=\left\{w_{r, s, t} \in \Omega \mid r+s+t\right.$ $=m\}$ is a basis for $H^{0}\left(F_{m}, \Omega^{1}\right)$ in the Hodge splitting of $H_{\mathrm{DR}}^{1}\left(F_{m}\right)$. 
The set of elements of $\Omega$ invariant under the action of $\Gamma_{a, b, c}^{m}$ descends to a basis of eigenforms for $H_{\mathrm{DR}}^{1}\left(J_{a, b, c}^{m}\right)$ under the action of $\mathrm{Z}\left[G_{m} / \Gamma_{a, b, c}^{m}\right]$. $\left(J_{a, b, c}^{m}\right)^{\text {new }}=J^{\text {new }}$ has CM (in the sense of Shimura-Taniyama) by the ring of integers

$$
\mathbf{Z}\left[G_{m} / \Gamma_{a, b, c}^{m}\right] /\left(f_{m}(g)\right) \approx \mathcal{O}_{K}
$$

of $K=\mathbf{Q}\left(\mu_{m}\right)$, with $\mathrm{CM}$ type

$$
H_{a, b, c}^{m}=\left\{h \in(\mathbf{Z} / m \mathbf{Z})^{*} \mid\langle h a\rangle+\langle h b\rangle+\langle h c\rangle=m\right\},
$$

where $\langle h\rangle$ denotes the unique representative of $h$ modulo $m$ between 0 and $m-1$.

Let $\mathscr{E}$ denote the set of positive integers $m$ which are different from each of the following numbers:

$2,3,4,6,8,9,10,12,14,15,18,20,21,22,24,26,28,30$,

$36,39,40,42,48,54,60,66,72,78,84,90,120,156,180$.

Then from the works of Koblitz-Rohrlich (for the cases where $m$ is relatively prime to 6) [8] and Aoki [1], for $m \in \mathscr{E}, J^{\text {new }}$ is non-simple if and only if

(1) $(a, b, c)$ is equivalent to $(1, r,-(1+r))$, where $1+r+r^{2} \equiv 0$ $(\bmod m)$, or

(2) $(a, b, c)$ is equivalent to $(1, s,-(1+s))$, where $s^{2} \equiv 1(\bmod m)$ and $s \not \equiv \pm 1(\bmod m)$, and $s \neq m / 2+1$ if $2^{3} \mid m$, or

(3) $(a, b, c)$ is equivalent to $(1,1,-2)$, with $2^{2} \mid m$, or

(4) $(a, b, c)$ is equivalent to $(1, m / 2+1, m / 2-2)$, with $2^{3} \mid m$.

In case (1), $J^{\text {new }}$ is isogeneous to a cube of an absolutely simple abelian variety. In cases (2) and (3), $J^{\text {new }}$ is isogeneous to a square of a simple abelian variety. Finally in case (4), $J^{\text {now }}$ is isogeneous to $X^{4}$ for some simple abelian variety $X$.

We shall denote $J^{\text {new }}$ by $A$ and $B$ in the first and second cases respectively.

Let $\rho$ be the automorphism of $F_{m}$ given by

$$
(X, Y, Z) \longrightarrow(Z, X, Y) \text {. }
$$

Let $\Gamma_{A}$ and $J_{A}$ denote the $\Gamma_{a, b, c}^{m}$ and $J_{a, b, c}^{m}$ associated with $A$. Since

$$
\rho \Gamma_{A} \rho^{-1} \subseteq \Gamma_{A}
$$

$\rho$ induces an automorphism of $G_{m} / \Gamma_{A}$ by conjugation. We note that $f_{m}\left(x^{l}\right)$ 
is divisible by $f_{m}(x)$ if $l$ and $m$ are relatively prime. Hence, if $g$ is a generator of $G_{m} / \Gamma_{A}$, then

$$
\rho f_{m}(g) J_{A}=f_{m}\left(\rho g \rho^{-1}\right) J_{A} \subseteq f_{m}(g) J_{A} .
$$

So $\rho$ induces an automorphism $\rho$ of $A$ such that the following diagram commutes:

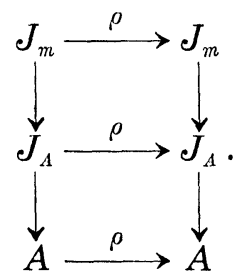

Let $\iota \in \operatorname{Aut}\left(F_{m}\right)$ be given by

$$
\text { ८: }(X, Y, Z) \longrightarrow(Y, X, Z) .
$$

Then we have a similar commutative diagram to the one above with $(A, \rho)$ replaced by $(B, \iota)$.

Since

$$
H^{1,0}\left(J^{\text {new }}, \mathbf{C}\right)=\oplus_{h \in H_{a, b, c}^{m}} V(\langle h a\rangle,\langle h b\rangle,\langle h c\rangle),
$$

where

$$
V(a, b, c)=\left\{\eta \in H^{1}\left(F_{m}, \mathbf{C}\right) \mid g^{*} \eta=\xi_{1}^{a} \xi_{2}^{b} \xi_{3}^{c} \eta \text { for all } g=\left(\xi_{1}, \xi_{2}, \xi_{3}\right) \in G_{m}\right\},
$$

a basis of holomorphic differential forms for $H^{0}\left(J^{\text {new }}, \Omega^{1}\right)$ is

$$
\left\{w_{\langle h a\rangle,\langle h b\rangle,\langle h c\rangle} \mid h \in H_{a, b, c}^{m}\right\} .
$$

The following lemma shows that the abelian varieties $A$ and $B$ are isogeneous to

$$
\prod_{l=0}^{2} A /\left\langle g_{l}\right\rangle \text { and } \prod_{l=0}^{1} B /\left\langle h_{l}\right\rangle
$$

respectively, where $g_{l}$ and $h_{l}$ denote $\sigma^{l} \rho \sigma^{-l}$ and $\sigma^{l} \iota \sigma^{-l}$ respectively.

Lemma 1.1. $H^{0}\left(J_{A}, \Omega^{1}\right)^{\left\langle g_{l}\right\rangle}$ is spanned by

$$
g_{l}^{*}\left\{w_{r, s} \mid w_{r, s} \in H^{0}\left(J_{A}, \Omega^{1}\right)\right\}
$$

and $H^{0}\left(J_{A}, \Omega^{1}\right)=\oplus_{l=0}^{2} H^{0}\left(J_{A}, \Omega^{1}\right)^{\langle g l\rangle}$. Similar statements hold for $H^{0}\left(J_{B}, \Omega^{1}\right)$, $h_{0}$ and $h_{1}$. 
Proof. Let $V_{l}$ and $W_{l}$ denote $\left(1+g_{l}+g_{l}^{2}\right)^{*} H^{0}\left(J_{A}, \Omega^{1}\right)$ and $H^{0}\left(J_{A}, \Omega^{1}\right)^{\left\langle g_{l}\right\rangle}$ respectively. Then $V_{l} \subseteq W_{l}$ and $\operatorname{dim} V_{l}=\operatorname{dim} H^{0}\left(J_{A}, \Omega^{1}\right) / 3$ by definition.

We claim that $W_{j} \cap\left(W_{k}+W_{l}\right)=\{0\}$ when $\{j, k, l\}=\{0,1,2\}$. We verify this for $j=0, k=1$ and $l=2$. The other cases are treated similarly.

Let $w_{0}=w_{1}+w_{2}$, where $w_{l} \in W_{l}(l=0,1,2)$. Then $w_{1}=\left(\sigma \rho \sigma^{-1}\right)^{*} w_{0}-$ $\left(\sigma \rho \sigma^{-1}\right)^{*} w_{2}=\left(\sigma^{-(r+2)}\right)^{*} w_{0}-\left(\sigma^{r+2}\right)^{*} w_{2}$. Therefore, $\left(\sigma^{-(r+2)}-1\right)^{*} w_{0}=\left(1-\sigma^{r+2}\right)^{*} w_{2}$. Applying $\left(\sigma^{r+2}\right)^{*}$ to both sides of the latter equation, we obtain $\left(1-\sigma^{r+2}\right)^{*}$ $\left(w_{0}-\left(\sigma^{r+2}\right)^{*} w_{2}\right)=0$. In particular,

$$
w_{0}-\left(\sigma^{r+2}\right)^{*} w_{2} \in H^{0}\left(F_{A} /\langle\sigma\rangle, \Omega^{1}\right) \approx H^{0}\left(\mathbf{P}^{1}, \Omega^{1}\right) .
$$

Hence, $w_{0}=\rho^{*} w_{0}=\rho^{*}\left(\sigma^{r+2}\right)^{*} w_{2}=\left(\sigma^{r+2} \rho\right)^{*} w_{2}=\left(\sigma^{2}\right)^{*}\left(\sigma^{2} \rho \sigma^{-2}\right)^{*} w_{2}=\left(\sigma^{2}\right)^{*} w_{2}$, and $\left(\sigma^{r}\right)^{*} w_{2}=w_{2}$. So, $w_{2}=0$, and $w_{0}=w_{1} \in W_{0} \cap W_{1}$, which we can show to be $\{0\}$, as before.

Let $A_{l}=A /\left\langle g_{l}\right\rangle$ and $B_{l}=B /\left\langle h_{l}\right\rangle$. Then each $A_{l}$ and $B_{l}$ is simple, and admits $\mathrm{CM}$ by the ring of integers in $L=K^{\langle r\rangle}$ and $M=K^{\langle s\rangle}$ respectively. To be precise, the endomorphisms $\sigma+\sigma^{r}+\sigma^{r^{2}}$ and $\sigma+\sigma^{s}$ of $A$ and $B$ descend to endomorphisms on $A_{0}$ and $B_{0}$ respectively. We identify the products $\prod_{l=0}^{2} A_{l}$ and $\prod_{l=0}^{1} B_{l}$ with $\left(A_{0}\right)^{3}$ and $\left(B_{0}\right)^{2}$ respectively through fixed isomorphisms $A_{l} \stackrel{\approx}{\longrightarrow} A_{0}$ and $B_{l} \stackrel{\approx}{\longrightarrow} B_{0}$.

Let us fix some terminology. (1) If $R$ is a ring, let $\Delta_{n}(R)$ be the subspace of the ring of $n \times n$-matrices $M_{n}(R)$ with entries in $R$ consisting of all the diagonal elements. If $\alpha_{1}, \cdots, \alpha_{n} \in R$, let $\Delta\left(\alpha_{1}, \cdots, \alpha_{n}\right)$ be the matrix $\left(\alpha_{i, j}\right)$ in $\Delta_{n}(R)$ with $\alpha_{i, j}=\delta_{i, j} \alpha_{j}$.

(2) If $X$ is an abelian variety, we associate to an endomorphism $\phi$ of $X^{n}$, the matrix $U_{\phi}$ in $M_{n}($ End $(X))$, if on points, $\phi:\left(\begin{array}{c}P_{1} \\ \vdots \\ \dot{P}_{n}\end{array}\right) \rightarrow U_{\phi} \cdot\left(\begin{array}{c}P_{1} \\ \vdots \\ \dot{P}_{n}\end{array}\right)$.

(3) Let $\phi: X \rightarrow Y$ be an isogeny of degree $N$. Let $\bar{\phi}: Y \rightarrow X$ be such that $\bar{\phi} \phi$ is multiplication by $N$ on $X$. Let $F_{\phi}: \operatorname{End}^{0}(X) \rightarrow \operatorname{End}^{0}(Y) \operatorname{map} \alpha$ in End $(X)$ to $N^{-1}(\phi \alpha \bar{\phi})$ in $\operatorname{End}^{0}(Y)$.

\section{§ 2. Rational endomorphisms}

Let $\Sigma_{l}$ be a basis for $H^{0}\left(A_{l}, \Omega^{1}\right)$ consisting of forms of the type $(1+$ $\left.g_{l}+g_{l}^{2}\right)^{*} w_{r, s}$. Then $\Sigma=\bigcup_{l=0}^{2} \Sigma_{l}$ is a basis for $H^{0}\left(A, \Omega^{1}\right)$. The main result in this section is 
Proposition 2.1. Let $m \in \mathscr{E}$. Then the following sequences are exact:

$$
\begin{aligned}
& 0 \longrightarrow\left(f_{m}(\sigma)\right) \longrightarrow \mathbf{Q}[\sigma, \rho] \longrightarrow \operatorname{End}^{0}(A) \longrightarrow 0, \\
& 0 \longrightarrow\left(f_{m}(\sigma)\right) \longrightarrow \mathbf{Q}[\sigma, \iota] \longrightarrow \operatorname{End}^{0}(B) \longrightarrow 0
\end{aligned}
$$

Proof. We will prove that $F: \mathbf{Q}[\sigma, \rho] \rightarrow \operatorname{End}^{0}\left(A_{0}^{3}\right)=M_{3}(L)$ is surjective. Since $f_{m}(\sigma) \in \operatorname{Ker}(F)$, a dimension argument shows that the first sequence is exact. We omit the proof of exactness of the second sequence.

The matrices for $\left(1+g_{l}+g_{l}^{2}\right)^{*}$ on $H^{0}\left(A, \Omega^{1}\right)$, with respect to the basis $\Sigma$ are:

$$
\left(\begin{array}{ccc}
3 & 0 & 0 \\
M_{0} & 0 & 0 \\
N_{0} & 0 & 0
\end{array}\right), \quad\left(\begin{array}{ccc}
0 & 3 & 0 \\
0 & M_{1} & 0 \\
0 & N_{1} & 0
\end{array}\right), \quad\left(\begin{array}{ccc}
0 & 0 & 3 \\
0 & 0 & M_{2} \\
0 & 0 & N_{2}
\end{array}\right)
$$

for $l=0,1,2$ respectively.

Now $w_{1, r} \in H^{0}\left(A, \Omega^{1}\right)$ and

$$
\left(1+g_{0}+g_{0}^{2}\right)^{*}\left(1+g_{1}+g_{1}^{2}\right)^{*} w_{1, r}=\left(1+\zeta^{r^{2+1}}+\zeta^{r^{2+2}}\right)\left(1+g_{0}+g_{0}^{2}\right)^{*} w_{1, r} .
$$

Let $l \in(\mathbf{Z} / m \mathbf{Z})^{*}-\left\{1,\left(r^{2}+1\right)\left(r^{2}+2\right)^{-1},\left(r^{2}+1\right)\left(r^{2}+2\right)^{-1}\right\}$. Since $\left\{\zeta^{a} \mid a \epsilon\right.$ $\left.(\mathbf{Z} / m \mathbf{Z})^{*}\right\}$ is a $\mathbf{Z}$-basis for $\mathcal{O}_{K}, \zeta^{r^{2+1}}, \zeta^{\left(r^{2}+1\right) l}, \zeta^{\left(r^{2}+2\right)}, \zeta^{\left(r^{2}+2\right) l}$ are linearly independent over $\mathbf{Q}$. Thus $\zeta^{r^{2+1}}+\zeta^{r^{2}+2}$ is not in $\mathbf{Q}$, and $1+\zeta^{r^{2+1}}+\zeta^{r^{2+2}} \neq 0$. This shows that the matrix $M_{0}$ is not the null matrix. In a similar way, we can prove that $N_{0}, M_{1}, N_{1}, M_{2}$ and $N_{2}$ are not zero. Then, in $\operatorname{End}\left(A_{0}^{3}\right)$ $=M_{3}\left(\mathcal{O}_{L}\right)$, the matrices for $\left(1+g_{l}+g_{l}^{2}\right)$ are:

$$
\left(\begin{array}{ccc}
3 & 0 & 0 \\
\alpha_{0} & 0 & 0 \\
\beta_{0} & 0 & 0
\end{array}\right), \quad\left(\begin{array}{lll}
0 & 3 & 0 \\
0 & \alpha_{1} & 0 \\
0 & \beta_{1} & 0
\end{array}\right), \quad\left(\begin{array}{ccc}
0 & 0 & 3 \\
0 & 0 & \alpha_{2} \\
0 & 0 & \beta_{2}
\end{array}\right)
$$

for $l=0,1,2$ respectively, where each $\alpha_{j}, \beta_{j}$ are in $\mathcal{O}_{L}$.

Let $X, Y, Z \in \mathbf{Q}[\sigma]$. In the group ring $\mathbf{Q}[\sigma, \rho]$, we have the following:

$$
\left(1+g_{l}+g_{l}^{2}\right)\left(X+\rho Y+\rho^{2} Z\right)=\left(1+g_{l}+g_{l}^{2}\right)\left(X+Y \sigma^{l\left(1-r^{2}\right)}+Z \sigma^{l(1-r)}\right)
$$

by using the relations $\rho \sigma \rho^{-1}=\sigma^{r}$ and $\rho^{-1} \sigma \rho=\sigma^{r^{2}}$ in $\operatorname{Aut}(A)$.

The determinant of the matrix $\left(\begin{array}{ccc}1 & 1 & 1 \\ 1 & \sigma^{1-r^{2}} & \sigma^{1-r} \\ 1 & \sigma^{2-2 r^{2}} & \sigma^{2-2 r}\end{array}\right)$ is $D=f(\sigma) \in \mathbf{Q}[\sigma]$, where

$$
f(x)=x^{\langle 4-r\rangle}-x^{\left\langle 4-r^{2}\right\rangle}+x^{\langle 1-r\rangle}-x^{\left\langle 1-r^{2}\right\rangle}+x^{\langle 2-2 r\rangle}-x^{\left\langle 2-2 r^{2}\right\rangle} \in \mathbf{Q}[x] .
$$

Since $r^{2}+r+1 \equiv 0(\bmod m)$, the exponents $4-r, 4-r^{2}, 1-r, 1-r^{2}$, $2-2 r, 2-2 r^{2}$ are pairwise distinct $(\bmod m)$ except possibly when $m \mid 3^{2}$ 
or $m=13$. Hence, $D \neq 0$ (the exceptional case $m=13$ is taken care of by inspection). In particular, there are $X, Y, Z \in \mathbf{Z}[\sigma]$ and a positive integer $N$ such that

$$
X+Y+Z=N D, \quad X+Y \sigma^{1-r^{2}}+Z \sigma^{1-r}=0, \quad X+Y \sigma^{2-2 r^{2}}+Z \sigma^{2-2 r}=0 .
$$

With the latter choice of $X, Y$ and $Z$, let the matrix of $\left(X+\rho Y+\rho^{2} Z\right)$ in $M_{3}\left(\mathcal{O}_{L}\right)$ be $\left(\alpha_{i, j}\right)$. From $\left(1+g_{1}+g_{1}^{2}\right)\left(X+\rho Y+\rho^{2} Z\right)=0$, we conclude that $\alpha_{2, j}=0$ for all $j$. On the other hand, $\alpha_{3, j}=0$ for all $j$, follows from $\left(1+g_{2}+g_{2}^{2}\right)\left(X+\rho Y+\rho^{2} Z\right)=0$. Then the matrix of $\left(X+\rho Y+\rho^{2} Z\right)(1+$ $\left.g_{0}+g_{0}^{2}\right)$ is

$$
\left(\begin{array}{ccc}
\alpha_{1,1} & \alpha_{1,2} & \alpha_{1,3} \\
0 & 0 & 0 \\
0 & 0 & 0
\end{array}\right) \cdot\left(\begin{array}{ccc}
3 & 0 & 0 \\
\alpha_{0} & 0 & 0 \\
\beta_{0} & 0 & 0
\end{array}\right)=\left(\begin{array}{ccc}
\delta_{0} & 0 & 0 \\
0 & 0 & 0 \\
0 & 0 & 0
\end{array}\right)
$$

where $\delta_{0}=3 \alpha_{1,1}+\alpha_{0} \alpha_{1,1}+\beta_{0} \alpha_{1,3} \in \mathcal{O}_{L}$.

Claim. $\quad \delta_{0} \neq 0$.

Suppose, on the contrary, that $\delta_{0}=0$. Then

$$
\begin{aligned}
N^{-1}\left(1+g_{0}+g_{0}^{2}\right)(X+\rho Y+ & \left.\rho^{2} Z\right)\left(1+g_{0}+g_{0}^{2}\right) \\
& =\left(1+\rho+\rho^{2}\right) D\left(1+g_{0}+g_{0}^{2}\right)=0 .
\end{aligned}
$$

We note that

$$
D^{*}\left(1+\rho+\rho^{2}\right)^{*} w_{1, r}=f(\zeta) w_{1, r}+f\left(\zeta^{r}\right) w_{r, m-r-1}+f\left(\zeta^{r^{2}}\right) w_{m-r-1,1}
$$

and if $\lambda_{m}=f\left(\zeta^{r}\right)+f\left(\zeta^{r}\right)+f\left(\zeta^{r^{2}}\right)$,

$$
\left(1+g_{0}+g_{0}^{2}\right)^{*} D^{*}\left(1+\rho+\rho^{2}\right)^{*}=\lambda_{m}\left(w_{1, r}+w_{r, m-r-1}+w_{m-r-1,1}\right) .
$$

We will show that $\lambda_{m} \neq 0$.

First, consider the prime case $m=p$. If $\lambda_{p}=0$, then the polynomial $g(x)=f(x)+f_{1}(x)+f_{2}(x)$, where $f_{j}(x)$ is the polynomial obtained by replacing each exponent $\langle a\rangle$ in $f(x)$ by $\left\langle a r^{j}\right\rangle$, has degree at most $p-1$, and $\zeta$ as a root. We note that $4-r, 4-r^{2}, 1-r, 1-r^{2}, 2-2 r, 2-2 r^{2}$ are distinct elements in $(\mathbf{Z} / p \mathbf{Z})^{*} /\left\{1, r, r^{2}\right\}$ for $p \neq 7,19,31$. Thus, with the above exceptions, $g(x) \neq 0$ and therefore, $g(x)= \pm f_{p}(x)$. This is a contradiction, since $g(1)=0$ but $f_{p}(1)=p$. Inspection shows that $\lambda_{p} \neq 0$ for $p=7,19,31$.

Now we treat the composite case.

Suppose that $l$ is a prime divisor of $m$ and $r-4$. Then $r^{2}+r+1$ $\equiv 0\left(\bmod l^{k}\right)$ and $r \equiv 4\left(\bmod l^{k}\right)$ imply that $l^{k} \mid 21$. Thus $\left(m, r^{2}-4\right) \mid 21$. 
Similarly $(m, r-4) \mid 21$. However, 7 can divide at most one of the two numbers $(m, r-4)$ and $\left(m, r^{2}-4\right)=(m, m-r-5)$. Furthermore, it is not difficult to verify that $(1-r, m)=\left(1-r^{2}, m\right) \mid 3$.

Case (1). First suppose that each of the integers $1-r, 1-r^{2}, 2-2 r$, $2-2 r^{2}$ are relatively prime to $m$ (this is the case when $(m, 6)=1$ ).

Case (1a). Both $(m, r-4)$ and $\left(m, r^{2}-4\right)$ are co-prime to 7 .

For $\beta \in K=\mathbf{Q}\left(\mu_{m}\right)$, let $\beta^{1+r+r^{2}}=\beta+\beta^{r}+\beta^{r^{2}}$, where $\left\{1, r, r^{2}\right\} \subseteq \operatorname{Gal}(K / \mathbf{Q})$. We note that if two of the integers $4-r, 4-r^{2}, 1-r, 1-r^{2}, 2-2 r$, $2-2 r^{2}$ represent the same class in $(\mathbf{Z} / m \mathbf{Z})^{*} /\left\{1, r, r^{2}\right\}$, then $m \in S$, where $S$ is a finite set of integers whose elements can be easily found using the congruence relation $r^{2}+r+1 \equiv 0(\bmod m)$. If $m \in S \cap \mathscr{E}$, inspection shows that $\lambda_{m} \neq 0$. If $m$ is not in $S$, a Z-basis for $\mathcal{O}_{L}$ is

$$
\left\{\zeta^{a\left(1+r+r^{2}\right)} \mid a \in(\mathbf{Z} / m \mathbf{Z})^{*} /\left\{1, r, r^{2}\right\}\right\},
$$

and we conclude that $\lambda_{m}$ is non-zero since it is a linear combination of elements of a subset of a Z-basis for $\mathcal{O}_{L}$.

Case (1b). $7 \|\left(m, r^{2}-4\right)$.

The elements of $\mathrm{Gal}(K / \mathbf{Q})$ which fix $\mathbf{Q}\left(\zeta^{7}\right)$ elementwise are the units $j \in(Z / m Z)^{*}$ such that $j \equiv 1(\bmod m / 7)$. We fix one such $j=1+k(m / 7) \neq 1$ in $(\mathbf{Z} / m \mathbf{Z})^{*}$. We make the following observation: if $a$ and $b j$ are equal in $(\mathbf{Z} / m \mathbf{Z})^{*} /\left\{1, r, r^{2}\right\}$, then $a \equiv r^{l} b j(\bmod m)$ implies $a \equiv r^{l} b(\bmod m / 7)$, and so $a$ and $b$ are equal in $(\mathbf{Z} /(m / 7) \mathbf{Z})^{*} /\left\{1, r, r^{2}\right\}$.

The calculations for case (1a) show that $1-r, 1-r^{2}, 2-2 r, 2-2 r^{2}$, $4-r$ are distinct in $(\mathbf{Z} / m \mathbf{Z})^{*} /\left\{1, r, r^{2}\right\}$ (hence in $\left.(\mathbf{Z} /(m / 7) \mathbf{Z})^{*} /\left\{1, r, r^{2}\right\}\right)$, except possibly when $m / 7 \in S$. For these exceptional values of $m, \lambda_{m} \neq 0$ by inspection. For the other values of $m$, the observation in the previous paragraph shows that $\bar{\lambda}_{m}=\lambda_{m}-\zeta^{\left(4-r^{2}\right)\left(1+r+r^{2}\right)}$ is such that $\bar{\lambda}_{m}^{j} \neq \bar{\lambda}_{m}$, since $\left\{\zeta^{a\left(1+r+r^{2}\right)} \mid a \in(\mathbf{Z} / m \mathbf{Z})^{*} /\left\{1, r, r^{2}\right\}\right\}$ is a $\mathbf{Z}$-basis for $\mathcal{O}_{L}$. Thus $\bar{\lambda}_{m} \notin \mathbf{Q}\left(\zeta^{7}\right)$, and $\lambda_{m}$ $\neq 0$.

Case (1c). $7 \|(m, r-4)$.

This is case (1b), with the roles of $r$ and $r^{2}$ reversed.

Case (2). Suppose now that $(1-r, m)=3$. If $m$ is odd, then we have that

$$
\begin{aligned}
& (1-r, m)=\left(1-r^{2}, m\right)=(2-2 r, m)=\left(2-2 r^{2}, m\right)=3 \\
& \quad \text { and } 9\left|(4-r, m) \cdot\left(4-r^{2}, m\right)\right| 9.7 .
\end{aligned}
$$


We apply the arguments in case (1) applied to $(1-r) / 3,\left(1-r^{2}\right) / 3,(2-$ $2 r) / 3,\left(2-2 r^{2}\right) / 3,(4-r) / 3,\left(4-r^{2}\right) / 3$ in $(\mathbf{Z} /(m / 3) \mathbf{Z}) * /\left\{1, r, r^{2}\right\}$.

If $m$ is even, we look at $(1-r) / 3,\left(1-r^{2}\right) / 3,(2-2 r) / 6,\left(2-2 r^{2}\right) / 6$, $(4-r) / 3,\left(4-r^{2}\right) / 3$ instead. The calculations are similar to the ones above.

This proves that $\lambda_{m} \neq 0$, and hence our claim that $\delta_{0} \neq 0$. We have shown that $F\left(\left(X+\rho Y+\rho^{2} Z\right)\left(1+g_{0}+g_{0}^{2}\right)\right)=\Delta\left(\delta_{0}, 0,0\right)$, with $\delta_{0} \neq 0$. Similarly, we can show the existence of $X_{l}, Y_{l}, Z_{l} \in \mathbf{Z}[\sigma]$ such that $\left(X_{l}+\rho Y_{l}\right.$ $\left.+\rho^{2} Z_{l}\right)\left(1+g_{l}+g_{l}^{2}\right)$ are mapped onto

$$
\Delta\left(0, \delta_{1}, 0\right) \text { and } \Delta\left(0,0, \delta_{2}\right) \quad \text { for } l=1,2 \text { respectively. }
$$

In particular, since $L \rightarrow \operatorname{End}^{0}\left(A_{0}^{3}\right)=M_{3}(L)$ (in which $\zeta^{1+r+r^{2}}$ is mapped to $\left.\left(\sigma+\sigma^{r}+\sigma^{r 2}\right)^{3}\right)$ is the diagonal embedding by the theory of complex multiplication, we conclude that

$$
\Delta_{3}(L) \subseteq \operatorname{Im}(F) \subseteq M_{3}(L) .
$$

We observe that

$$
\begin{aligned}
& \sigma^{*}\left(\sigma\left(1+\rho+\rho^{2}\right) \sigma^{-1}\right)^{*} w_{a, b}=\left(1+\rho+\rho^{2}\right)^{*} \sigma^{*} w_{a, b}, \quad \text { and } \\
& \sigma^{*}\left(\sigma^{2}\left(1+\rho+\rho^{2}\right) \sigma^{-2}\right)^{*} w_{a, b}=\left(\sigma\left(1+\rho+\rho^{2}\right) \sigma^{-1}\right)^{*} \sigma^{*} w_{a, b} .
\end{aligned}
$$

Thus the matrix for $\sigma$ in $M_{3}\left(\mathcal{O}_{L}\right)$ is of the form: $\left(\begin{array}{lll}a & b & c \\ d & 0 & 0 \\ 0 & e & 0\end{array}\right)$, for some $a$, $b, c, d$ and $e$ in $\mathcal{O}_{L}$ with $c d e \in\left(\mathcal{O}_{L}\right)^{*}$ (this follows from $\operatorname{det}(\sigma)^{m}=1$ ). Therefore the image of $F$ contains the following matrices:

$$
\left(\begin{array}{lll}
0 & b & c \\
d & 0 & 0 \\
0 & e & 0
\end{array}\right), \quad\left(\begin{array}{ccc}
b d & c e & 0 \\
0 & b d & c d \\
d e & 0 & 0
\end{array}\right)=\left(\begin{array}{ccc}
0 & b & c \\
d & 0 & 0 \\
0 & e & 0
\end{array}\right)^{2}, \quad\left(\begin{array}{ccc}
0 & c e & 0 \\
0 & 0 & c d \\
d e & 0 & 0
\end{array}\right)
$$

This completes the proof that $F$ is surjective.

\section{§ 3. Homology groups}

Let $I:[0,1] \rightarrow F_{m}(\mathbf{C})$ denote the one-simplex

$$
I(t)=\left(t^{1 / m},(1-t)^{1 / m}, \alpha\right), \quad t \in[0,1],
$$

where $\alpha=-1$ if $m$ is odd and a primitive $2 m$-th root of unity if $m$ is even. Let $g$ be the one-cycle:

$$
\begin{array}{ll}
g=(\sigma \tau)^{(m-1) / 2}(1-\sigma)(1-\tau) I & \text { if } m \text { is odd, and } \\
g=\left(1-\sigma^{-1}\right)\left(1-\tau^{-1}\right) I & \text { if } m \text { is even. }
\end{array}
$$


The homology group $H_{1}\left(F_{m}(\mathbf{C}), \mathbf{Z}\right)$ is generated by $g$ [11]. Moreover by the period calculations in [11], we have that $\rho(g)=g$ and $\iota(g)=-g$ [9].

Proposition 3.1. $H_{1}\left(F_{m}(\mathbf{C}), \mathbf{Z}\right)$ is a cyclic $\mathbf{Z}\left[G_{m}\right]$-module, with g as a generator such that $\rho(g)=g$ and $\iota(g)=-g$ in homology.

For the rest of this paper, let $p$ be a fixed prime congruent to 1 $(\bmod 6)$, let $r$ be a fixed cube root of unity modulo $p, K=\mathbf{Q}\left(\mu_{p}\right), \zeta$ be a fixed $p$-th root of unity, and $A$ be the Jacobian variety of the curve $F_{A}$ :

$$
y^{p}=x(1-x)^{r} .
$$

$A$ has $\mathrm{CM}$ by $\mathcal{O}_{K}$ : we fix the embedding

$$
\mathcal{O}_{K} \longrightarrow \operatorname{End}_{K}(A), \quad \zeta \longrightarrow \sigma=(\zeta, 1,1) \text {. }
$$

Let $\varphi_{A}: F_{p} \rightarrow F_{A}$ denote the canonical projection, and let $I_{A}$ be the one simplex $\varphi_{A} I$ on $F_{A}$. Fix a base point $e_{0}$ in $F_{p}(\mathbf{C})$, and let $x_{0}$ be its image in $F_{A}(\mathbf{C})$ under $\varphi_{A}$. The cyclic covering $\varphi_{A}$ gives rise to a monomorphism

$$
H=\pi_{1}\left(F_{p}(\mathbf{C}), e_{0}\right) \longrightarrow \pi_{1}\left(F_{A}(\mathbf{C}), x_{0}\right)=G
$$

of fundamental groups. $G / H$ is a cyclic group of order $p$ since $\varphi_{A}$ has degree $p$. So $H$ contains the commutator subgroup of $G$, and the homomorphism

$$
H_{1}\left(F_{p}\right)=H_{1}\left(F_{p}(\mathbf{C}), \mathbf{Z}\right) \longrightarrow H_{1}\left(F_{A}(\mathbf{C}), \mathbf{Z}\right)=H_{1}\left(F_{A}\right)
$$

factors as follows:

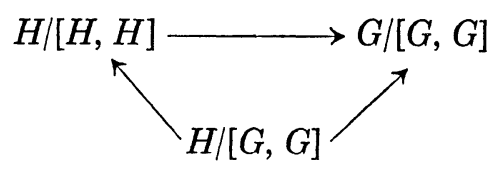

Thus, the index of the image $T$ of $H_{1}\left(F_{p}\right)$ in $H_{1}\left(F_{A}\right)$ is $p . \quad T$, by definition, is a cyclic $\mathrm{Z}[\sigma]$-module with $(\sigma-1)\left(\sigma^{r}-1\right) I_{A}$ as a generator by Proposition 3.1 .

Let $\bar{T}$ be the $\mathbf{Z}[\sigma]$-submodule of $H_{1}\left(F_{A}\right)$ generated by $\alpha=(\sigma-1) I_{A}$. Then $T \subseteq \bar{T} \subseteq H_{1}\left(F_{A}\right)$. We claim that $T \neq \bar{T}$, from which it follows that $H_{1}\left(F_{A}\right)=\bar{T}$.

Identifying

$$
\mathbf{Q}[\sigma] /\left(f_{p}(\sigma)\right) \stackrel{\approx}{\longrightarrow}, \quad \sigma \longrightarrow \zeta,
$$


$H_{1}\left(F_{A}\right) \otimes \mathbf{Q}$ is a vector space over $K$. Hence the annihilator of $H_{1}\left(F_{A}\right) \otimes \mathbf{Q}$ as a $\mathbf{Q}[\sigma]$-module is $\left(f_{p}(\sigma)\right)$, and the annihilator of $H_{1}\left(F_{A}\right)$, as a $\mathbf{Z}[\sigma]$ module is

$$
\left(f_{p}(\sigma)\right) \mathbf{Q}[\sigma] \cap \mathbf{Z}[\sigma]=\left(f_{p}(\sigma)\right) \mathbf{Z}[\sigma] .
$$

Since $H_{1}\left(F_{A}\right)$ is torsion-free over $\mathbf{Z}$, and $\left[H_{1}\left(F_{A}\right): \bar{T}\right]<\infty, \operatorname{Ann}_{\mathbf{Z}[\sigma]}(\bar{T})=$ $\left(f_{p}(\sigma)\right) \mathbf{Z}[\sigma]$.

Suppose, on the contrary, that $T=\bar{T}$. Then $\alpha=a(\sigma)(\sigma-1) \alpha$ for some $a(x) \in \mathbf{Z}[x]$. Therefore, $(a(\sigma)(\sigma-1)-1) \alpha=0$ implies $a(x)(x-1)-1$ $=b(x) f_{p}(x)$ for some $b(x) \in \mathbf{Z}[x]$. Then $-1=b(1) p$ in $\mathbf{Z}$, a contradiction. Thus, $H_{1}\left(F_{A}\right)=\bar{T}$.

Let $\bar{I}=\rho I$ and $\bar{I}_{A}=\varphi_{A} \bar{I}$. From $\rho(g)=g$ in $H_{1}\left(F_{p}\right)$, we obtain

$$
(\sigma-1)\left(\sigma^{r}-1\right) I_{A}=\sigma^{1+r((p+1) / 2)}\left(\sigma^{r}-1\right)\left(\sigma^{p-r-1}-1\right) \bar{I}_{A}
$$

in $H_{1}\left(F_{A}\right)$.

Let $v \in H_{1}\left(F_{A}\right)$ be such that $\left(\sigma^{r}-1\right) v=0$. Passing to $\mathcal{O}_{K} \subseteq \operatorname{End}_{K}(A)$, we have $\left(\zeta^{r}-1\right) v=0$. Then $p v= \pm N_{\mathbf{Q}}^{K}\left(\zeta^{r}-1\right) v=0$, and $v=0$. Thus, we have proved

Proposition 3.2. $H_{1}\left(F_{A}\right)$ is a cyclic $\mathcal{O}_{K}$-module with $g_{A}=(1-\sigma) I_{A}$ as a generator. Moreover,

$$
\rho\left(g_{A}\right)=\zeta^{r((p-1) / 2)}\left(\frac{\zeta^{r}-1}{\zeta^{r^{2}}-1}\right) g_{A} .
$$

\section{§4. Endomorphisms}

In the present section, we prove the following theorem. Let $\pi=\zeta$ $-1 \in \mathbf{Z}[\zeta] \subseteq$ End $(A)$ and $W=p^{-1}\left(1+r \rho+r^{2} \rho^{2}\right)(\sigma-1)^{p-3} \in \mathbf{Q}[\sigma, \rho]$.

Theorem 4.1. End $(A)=\operatorname{Im}(\mathbf{Z}[\sigma, \rho, W])$ has group index $p^{3}$ over $\operatorname{Im}(\mathbf{Z}[\sigma, \rho])$.

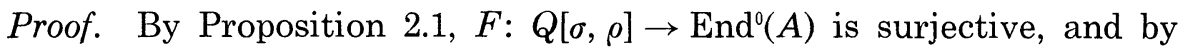
Proposition 3.2, $H_{1}\left(F_{A}\right)$ is a cyclic $\mathbf{Z}[\zeta]$-module with a generator $g_{A}$ such that $\rho\left(g_{A}\right)=\eta g_{A}, \rho^{2}\left(g_{A}\right)=\xi g_{A}$, where

$$
\eta=\zeta^{r((p-1) / 2)-1} \frac{\left(\zeta^{r}-1\right)}{\left(\zeta^{2}-1\right)} \text { and } \xi=\zeta^{r^{2+(p+1) / 2}} \frac{\left(\zeta^{r}-1\right)}{(\zeta-1)} .
$$

We will use the following to determine End $(A)$ :

$$
\text { End }(A)=\left\{\alpha \in \operatorname{End}^{\circ}(A) \mid \alpha\left(H_{1}\left(F_{A}\right)\right) \subseteq H_{1}\left(F_{A}\right)\right\} .
$$


Let $X, Y, Z \in K$. Then $\alpha=X+Y \rho+Z \rho^{2} \in \operatorname{End}(A)$ if and only if $\alpha\left(\zeta^{a} g_{A}\right)$ $\subseteq H_{1}\left(F_{A}\right)$ for all $a \in \mathbf{Z}$, or equivalently, for all $a \in \mathbf{Z}$,

$$
X \zeta^{a}+Y \zeta^{a r} \eta+Z \zeta^{-a(r+1)} \xi \in \mathbf{Z}[\zeta] .
$$

Let $\tilde{X}=X, \tilde{Y}=Y_{\eta}$ and $\tilde{Z}=Z \xi$. Then (4.1) reads as

$$
\tilde{X} \zeta^{a}+\tilde{Y} \zeta^{a r}+\tilde{Z} \zeta^{-a(r+1)} \in \mathbf{Z}[\zeta] .
$$

Using $\tilde{X}+\tilde{Y}+\tilde{Z} \in \mathbf{Z}[\zeta]$ and (4.2) to eliminate $\tilde{X}$, we obtain for all $a \in$ $(\mathbf{Z} / p \mathbf{Z})^{*}$,

$$
\tilde{Y}\left(\zeta^{a r}-\zeta^{a}\right)+\tilde{Z}\left(\zeta^{-a(r+1)}-\zeta^{a}\right) \in \mathbf{Z}[\zeta] .
$$

For such $a, \zeta^{a r}-\zeta^{a}$ and $\zeta^{-a(r+1)}-\zeta^{a}$ are elements of the ideal $(\pi)$ of $Z[\zeta]$.

Let $D_{a, b}$ be the determinant of the following matrix:

$$
\left(\begin{array}{ll}
\zeta^{a r}-\zeta^{a} & \zeta^{-a(r+1)}-\zeta^{a} \\
\zeta^{b r}-\zeta^{b} & \zeta^{-b(r+1)}-\zeta^{b}
\end{array}\right)
$$

Then

$$
D_{a, b}=\left\{\zeta^{a r-b(r+1)}+\zeta^{b r+a}+\zeta^{b-a(r+1)}\right\}-\left\{\zeta^{a r+b}+\zeta^{a-b(r+1)}+\zeta^{b r-a(r+1)}\right\},
$$

and (4.3) implies that

$$
D_{a, b} \tilde{Y}, \quad D_{a, b} \tilde{Z} \in(\pi)
$$

for all $a, b \in(\mathbf{Z} / p \mathbf{Z})^{*}$.

If we set $(a, b)=(r+1,1)$ and $(a, b)=(1,-r)$ in (4.4), we obtain, after simplification,

$$
\left(\zeta^{3 r+3}+\zeta^{3}+1-3 \zeta^{r+2}\right) \tilde{Z} \in(\pi) \text { and }\left(\zeta^{3 r+3}+\zeta^{3 r}+1-3 \zeta^{2 r+1}\right) \tilde{Z} \in(\pi)
$$

respectively. By subtracting one from the other, we obtain

$$
\zeta^{3}\left(\zeta^{r-1}-1\right)^{2} \tilde{Z} \in(\pi) \text {. }
$$

Since $(p, r-1)=1, \pi^{2} \tilde{Z} \in \mathbf{Z}[\zeta]$. By symmetry, $\pi^{2} \tilde{Y} \in \mathbf{Z}[\zeta]$.

We write $Y_{0}=\tilde{Y} \pi^{2}$ and $Z_{0}=\tilde{Z} \pi^{2}$. Then $Y_{0}, Z_{0} \in \mathbf{Z}[\zeta]$, and (4.3) can be rewritten as

$$
Y_{0} \frac{\left(\zeta^{r}-\zeta\right)^{h}}{(\zeta-1)^{2}}+Z_{0} \frac{\left(\zeta^{-(r+1)}-\zeta\right)^{h}}{(\zeta-1)^{2}} \in \mathbf{Z}[\zeta]
$$

where $h$ ranges over $H=\operatorname{Gal}(K / \mathbf{Q})$, or equivalently,

$$
Y_{0}+\varepsilon_{h} \cdot Z_{0} \in(\pi) \quad \text { for all } h \in H \text {, }
$$


where

$$
\varepsilon_{h}=\frac{\left(\zeta^{r^{2}}-\zeta\right)^{h}}{\left(\zeta^{r}-\zeta\right)^{h}}=\left(\sum_{\jmath=0}^{r} \zeta^{j(r-1)}\right)^{h} \in(\mathbf{Z}[\zeta])^{*}
$$

Clearly, (4.5) may be rewritten as

$$
Y_{0} \equiv r^{2} Z_{0}(\bmod \pi) \text {. }
$$

We have proved that $\alpha=X+Y \rho+Z \rho^{2}$ is in $\operatorname{End}(A)$ if and only if (*) $X+n Y+\xi Z \in \mathbf{Z}[\zeta]$, and

(**) $Y_{0} \equiv r^{2} Z_{0}(\bmod \pi)$, where $Y_{0}=\pi^{2} \eta Y$ and $Z_{0}=\pi^{2} \xi Z$.

We write

$$
Y_{0} \equiv a_{0}+a_{1} \pi\left(\bmod \pi^{2}\right), \quad Z_{0} \equiv b_{0}+b_{1} \pi\left(\bmod \pi^{2}\right),
$$

where $a_{0}, a_{1}, b_{0}, b_{1} \in \mathbf{Z}$. By $(* *), a_{0} \equiv r^{2} b_{0}(\bmod p)$. Thus, we find that $\alpha$ is congruent to

$$
\begin{aligned}
b_{0} \frac{1}{\pi^{2}}\left\{-\left(r^{2}+1\right)+r^{2} \eta^{-1} \rho+\xi^{-1} \rho^{2}\right\}+ & a_{0} \frac{1}{\pi}\left(-1+\eta^{-1} \rho\right) \\
& +b_{1} \frac{1}{\pi}\left(-1+\xi^{-1} \rho^{2}\right)
\end{aligned}
$$

modulo $\operatorname{Im}(\mathbf{Z}[\sigma, \rho])$.

By inspection,

$$
\begin{aligned}
& v_{0}=\frac{1}{\pi^{2}}\left\{-\left(r^{2}+1\right)+r^{2} \eta^{-1} \rho+\xi^{-1} \rho^{2}\right\}, \\
& v_{1}=\frac{1}{\pi}\left(-1+\eta^{-1} \rho\right), \quad v_{2}=\frac{1}{\pi}\left(-1+\xi^{-1} \rho^{2}\right)
\end{aligned}
$$

satisfy $(*)$ and $(* *)$. Hence, they are in $\operatorname{End}(A)$, and we conclude that

$$
\text { End }(A)=\operatorname{Im}(\mathbf{Z}[\sigma, \rho])+Z v_{0}+Z v_{1}+Z v_{2} .
$$

From (4.8), the quotient group

$$
Q=\operatorname{End}(A) / \Lambda \quad \text { where } \Lambda=\operatorname{Im}(\mathbf{Z}[\sigma, \rho])
$$

is an elementary $p$-abelian group. So $Q$ is an $\mathbf{F}_{p}$-vector space, and $\operatorname{dim}_{\mathbf{F}_{p}}(Q) \leq 3$.

The theorem follows from the next few lemmas.

LEMMA 4.2. Let

$$
w=\left(1+r \rho+r^{2} \rho^{2}\right) \frac{1}{\pi^{2}}=\frac{1}{\pi^{2}}+\frac{r}{\left(\zeta^{r}-1\right)^{2}} \rho+\frac{r^{2}}{\left(\zeta^{r^{2}}-1\right)^{2}} \rho^{2} \in \operatorname{End}^{0}(A) .
$$


Then $w \in \operatorname{End}(A)$.

Proof. We verify (**) for $w$. We have $Y_{0}=\left(r \pi^{2} \eta\right) /\left(\zeta^{r}-1\right)^{2}$ and $Z_{0}=$ $\left(r^{2} \pi^{2} \xi\right) /\left(\zeta^{2}-1\right)^{2}$ in the notation of the proof of Theorem 4.1. Since

$$
Y_{0} \equiv r \zeta^{r(p-1) / 2-1} \frac{(\zeta-1)}{\left(\zeta^{r}-1\right)} \frac{(\zeta-1)}{\left(\zeta^{r^{2}}-1\right)} \equiv r(\bmod \pi)
$$

and

$$
Z_{0} \equiv r^{2} \zeta^{r^{2}+(p+1) / 2} \frac{(\zeta-1)}{\left(\zeta^{r^{2}}-1\right)} \frac{\left(\zeta^{r}-1\right)}{\left(\zeta^{r^{2}}-1\right)} \equiv r^{2}(\bmod \pi)
$$

we have $Y_{0} \equiv r^{2} Z_{0}(\bmod \pi)$. Likewise, $(*)$ can be verified for $w$. This completes the proof of the lemma.

Lemma 4.3. Let $\Sigma=\operatorname{Im}(\mathbf{Z}[\sigma, \rho, W])$. Then $\Sigma \subseteq \operatorname{End}(A)$, and the following are elements of $\Sigma$ :

$$
w, w_{0}=\{1+(r+1) \rho\} \frac{1}{\pi}, \quad w_{1}=\left(r \rho-\rho^{2}\right) \frac{1}{\pi} .
$$

Proof. Let $u \in(\mathbf{Z}[\zeta])^{*}$ be the endomorphism of $A$ such that $p=u \pi^{p-1}$. As an element of $\operatorname{End}^{\circ}(A), W=w u^{-1}$. Hence the image of $w$ is in $\Sigma$, and $\Sigma \subseteq$ End $(A)$.

From $w \sigma=\left(\sigma+r \sigma^{r} \rho+r^{2} \sigma^{r^{2}} \rho^{2}\right) 1 / \pi^{2}$ and $\sigma w=\left(\sigma+r \sigma \rho+r^{2} \sigma \rho^{2}\right) 1 / \pi^{2}$, we have

$$
\sigma w-w \sigma \equiv(r-1) \rho\{1+(r+1) \rho\} \frac{1}{\pi}(\bmod \Lambda)
$$

Since $p$ does not divide $r-1$ and $\rho \in \operatorname{Aut}(A)$, there is a $\lambda \in \mathbf{Z}$ such that

$$
\{1+(r+1) \rho\} \frac{1}{\pi} \equiv \lambda \rho^{2}(\sigma w-w \sigma)(\bmod \Lambda) .
$$

Hence, $w_{0} \in \Sigma$. Since $w_{1} \equiv r \rho w_{0}(\bmod \Lambda)$, we have $w_{1} \in \Sigma$ also.

LEMma 4.4. The mapping $f:(Z[\zeta])^{3} \rightarrow \Lambda,(X, Y, Z) \rightarrow X+\rho Y+\rho^{2} Z$ is a right $\mathbf{Z}[\zeta]$-module isomorphism.

Proof. By definition, $f$ is surjective. By Proposition 2.1, $f \otimes 1: K^{3}=$ $\left(\mathbf{Q}\left(\mu_{p}\right)\right)^{3} \rightarrow \Lambda \otimes \mathbf{Q}$ is an isomorphism. Hence $f$ is injective.

Lemma 4.5. Let $V$ be the subspace of $Q$ spanned by $w, w_{0}$ and $w_{1}$. Then $\operatorname{dim}_{\mathbf{F}_{p}}(V)=3$. 
Proof. Let $\lambda, \lambda_{0}, \lambda_{1} \in \mathbf{Z}$ be such that

$$
\lambda w+\lambda_{0} w_{0}+\lambda_{1} w_{1} \in \Lambda .
$$

Multiplying by $\pi$ on the right, $\lambda\left(1+r \rho+r^{2} \rho^{2}\right) \in \pi \Lambda$. Using Lemma 4.4, $\lambda / \pi \in \mathbf{Z}[\zeta]$. Hence $\lambda \in(\pi) \cap \mathbf{Z}=p \mathbf{Z}$. Since $p / \pi^{2} \in \mathbf{Z}[\zeta]$, we have

$$
\lambda_{0} w_{0}+\lambda_{1} w_{1} \in \Lambda \text {. }
$$

Another application of Lemma 4.4 to (4.10) gives $\lambda_{0}, \lambda_{1} \in p \mathbf{Z}$. Therefore $\left\{w, w_{0}, w_{1}\right\}$ is an $\mathbf{F}_{p}$-basis for $V$.

Combining Lemmas 4.3 and 4.5,

$$
\operatorname{dim}_{\mathbf{F}_{p}}(\Sigma / \Lambda) \geq 3
$$

Since $\operatorname{dim}_{\mathrm{F}_{p}}(Q) \leq 3$, we have the desired equality: $\operatorname{End}(A)=\Sigma$, and $\operatorname{End}(A)$ has group index $p^{3}$ over 1 . This completes the proof of Theorem 4.1.

Corollary 4.6. A free Z-basis for $\operatorname{End}(A)$ is given by:

$$
\left\{\rho^{i} \pi^{k} \mid 0 \leq j \leq 2,0 \leq k \leq p-4\right\} \cup\left\{\rho \pi^{p-3}, \rho^{2} \pi^{p-3}, \rho \pi^{p-2}\right\} \cup\left\{w, w_{0}, w_{1}\right\} .
$$

Proof. Let $M$ be the Z-submodule of End $(A)$ spanned by the above elements. Inspection shows that $\Lambda \subseteq M$. By Lemma 4.5, the corollary follows.

Remarks. Let $k$ be a proper subfield of $K$, and let $h$ be a generator of $\operatorname{Gal}(K / k) \subseteq(\mathbf{Z} / p \mathbf{Z})^{*}$. Then the subring of endomorphisms of $A$ defined over $k$ is

$$
\operatorname{End}(A)=\operatorname{Im}\left(\mathbf{Z}\left[\sum_{j=1}^{t-1} \sigma^{a h^{j}}, \rho \mid a \in \mathbf{Z}\right]\right),
$$

where $t$ is the order of $h$. $\operatorname{End}_{k}(A)$ is commutative if and only if $k$ is $\mathbf{Q}$ or $L=K^{\langle r\rangle}$. In the latter cases, $\operatorname{End}_{k}(A)$ are contained in $\mathbf{Z} \times \mathbf{Z}[(1+\sqrt{-3}) / 2]$ and $\mathcal{O}_{K} \times \mathcal{O}_{K(\sqrt{-3})}$ respectively.

\section{$\S 5$. Action of rho on some division points}

Let $P_{1}, P_{2}$ and $P_{3}$ be any 3 points on $F_{p}$ where $X=0, Y=0$ and $Z=0$ respectively. Recall that $\varphi_{A}: F_{p} \rightarrow F_{A}$ is the canonical projection. Set

$$
\infty_{2}=\varphi_{A}\left(P_{1}\right), \quad \infty_{3}=\varphi_{A}\left(P_{2}\right), \quad \text { and } \quad \infty_{1}=\varphi_{A}\left(P_{3}\right)
$$

Then the group of $A[\pi]$ of $\pi$-division points on $A$ has order $p$, and con- 
tains all the divisor classes of degree zero supported on the set of cusps $\left\{\infty_{1}, \infty_{2}, \infty_{3}\right\}$ of $F_{A}$.

For each integer $a \geq 1$,

$$
\pi^{a} \rho=\rho\left(\zeta^{r^{2}}-1\right)^{a}=\rho \frac{\zeta^{r^{2}}-1}{\zeta-1} \pi^{a}
$$

in End $(A)$, so that $\rho$ induces an automorphism of $A\left[\pi^{a}\right]$ by restriction.

LEMMA 5.1. $\rho$ acts on $A[\pi]$ as multiplication by $r$.

Proof. Recall that the equation of $F_{A}$ is $v^{p}=u(1-u)^{r}$. The divisor of the rational function $v$ on $F_{A}$ is $\infty_{2}-(r+1) \infty_{1}+r \infty_{3}$. Hence, on $A$, $\infty_{2}-(r+1) \infty_{1}+r \infty_{3}=0=\infty_{1}-(r+1) \infty_{3}+r \infty_{2}$ (the latter equality is obtained by applying $\rho$ to the former). In particular,

$$
\rho\left(\infty_{1}-\infty_{2}\right)=\infty_{2}-\infty_{3}=(r+1)\left(\infty_{1}-\infty_{3}\right)=r\left(\infty_{1}-\infty_{2}\right) .
$$

Lemma 5.2. There is an element $Q \in A\left[\pi^{2}\right]-A[\pi]$ such that $\rho(Q)=Q$.

Proof. Let us fix a $Q$ in $A\left[\pi^{2}\right]-A[\pi]$. Then $A\left[\pi^{2}\right]=\{(a+b \pi) Q \mid a, b$ $\left.\in \mathbf{F}_{\rho}\right\}$ is a vector space of dimension 2 over $\mathbf{F}_{p}$. Let $f(x)$ be the minimal polynomial of $\rho$ restricted to $A\left[\pi^{2}\right]$. Since $\rho$ has order 3 , we have $f(x) \mid(x-1)(x-r)\left(x-r^{2}\right)$ in $\mathbf{F}_{p}[x]$. Since $\rho$ can have at most two distinct eigenvalues, and $f(x)$ splits completely, we have $f(x)=x-\lambda_{1}$ or $f(x)=$ $\left(x-\lambda_{1}\right)\left(x-\lambda_{2}\right)$, where $\lambda_{1}, \lambda_{2} \in\left\{1, r, r^{2}\right\}$ and $\lambda_{1} \neq \lambda_{2}$.

Suppose that $f(x)=x-\lambda_{1}$. Then $\lambda_{1}(\pi Q)=\rho(\pi Q)=\left(\zeta^{r}-1\right) \pi Q=$ $\lambda_{1}\left\{\left(\zeta^{r}-1\right) / \pi\right\} \pi Q=\lambda_{1}\{r+(r(r-1) / 2) \pi+\cdots\} \pi Q=\lambda_{1} r(\pi Q)$, whence $\lambda_{1}=\lambda_{1} r$ and $\lambda_{1}=0$, a contradiction. Hence, $f(x)=\left(x-\lambda_{1}\right)\left(x-\lambda_{2}\right)$, and there is an $\mathbf{F}_{p}$-basis $Q_{1}, Q_{2}$ of $A\left[\pi^{2}\right]$ such that the matrix of $\rho$ with respect to $\left\{Q_{1}, Q_{2}\right\}$ is $\left(\begin{array}{cc}\lambda_{1} & 0 \\ 0 & \lambda_{2}\end{array}\right)$. Since at least one of $Q_{1}, Q_{2}$ is not in $A[\pi]$, we have found a $Q$ in $A\left[\pi^{2}\right]-A[\pi]$ and a $\lambda \in\left\{1, r, r^{2}\right\}$ such that $\rho(Q)=\lambda Q$. By Lemma $5.1, r(\pi Q)=\rho(\pi Q)=\lambda r(\pi Q)$, and $\lambda=1$. This completes the proof of the lemma.

Remarks. (1) In the same way as above, we can show that there is a $Q \in A\left[\pi^{3}\right]-A\left[\pi^{2}\right]$ such that $\rho(Q)=r^{2} Q$. We also remark that the annihilator, in $\operatorname{End}(A)$, of $A[\pi]$ is

$$
\mathbf{Z}[\zeta] \pi+\mathbf{Z}[\zeta](\rho-r)+\mathbf{Z}[\zeta]\left(\rho^{2}-r^{2}\right)+\mathbf{Z}\left(1+r \rho-(r+1) \rho^{2}\right) \frac{1}{\pi} .
$$


(2) If - denotes complex conjugation, then for $Q \in A\left[\pi^{2}\right]-A[\pi], \bar{Q}=$ $-Q \Leftrightarrow \rho(Q)=Q$.

\section{$\S 6$. The kernel of an isogeny}

Let $X_{j}=F_{A} \mid\left\langle\sigma^{j} \rho \sigma^{j}\right\rangle,(j=0,1,2)$, and we denote the canonical projection $F_{A} \rightarrow X_{j}$ by $\varphi_{j}$. Let $\varphi$ be the isogeny

$$
\varphi=\prod_{j=0}^{2}\left(\varphi_{j}\right)_{*}: A \longrightarrow \prod_{j=0}^{2} \operatorname{Jac}\left(X_{j}\right)
$$

Lemma 6.1. $\operatorname{Ker}(\varphi) \subseteq A\left[\pi^{2}\right]$

Proof. The composition $A \stackrel{\left(\varphi_{j}\right)_{*}}{\longrightarrow} \operatorname{Jac}\left(X_{j}\right) \stackrel{\left(\varphi_{j}\right)^{*}}{\longrightarrow} A$ is $\zeta^{j}\left(1+\rho+\rho^{2}\right) \zeta^{-j} \epsilon$ End $(A)$, so that $\operatorname{Ker}\left(\varphi_{\jmath}\right)_{*} \subseteq A\left[\zeta^{\jmath}\left(1+\rho+\rho^{2}\right) \zeta^{-j}\right]$. Let $N$ be $\bigcap_{j=0}^{2} A\left[\zeta^{\jmath}(1+\right.$ $\left.\left.\rho+\rho^{2}\right) \zeta^{-j}\right]$. Then

$$
\operatorname{Ker}(\varphi)=\operatorname{Ker}\left(\varphi_{0}\right)_{*} \cap \operatorname{Ker}\left(\varphi_{1}\right)_{*} \cap \operatorname{Ker}\left(\varphi_{2}\right)_{*} \subseteq N .
$$

We claim that $N \subseteq A\left[\pi^{2}\right]$. Let $D \in N$. Then we have

$$
\begin{gathered}
\left(1+\rho+\rho^{2}\right) D=0, \\
\left(1+\zeta^{1-r} \rho+\zeta^{1-r^{2}} \rho^{2}\right) D=0,
\end{gathered}
$$

and

$$
\left(1+\zeta^{2-2 r} \rho+\zeta^{2-2 r^{2}} \rho^{2}\right) D=0,
$$

using the relations $\rho \sigma \rho^{-1}=\sigma^{r}$ and $\rho^{-1} \sigma \rho=\sigma^{r 2}$ in Aut $\left(F_{A}\right)$. From (6.1) and (6.2), we obtain that

$$
\left\{\left(\zeta^{1-r^{2}}-1\right)+\left(\zeta^{1-r^{2}}-\zeta^{1-r}\right) \rho\right\} D=0 .
$$

From (6.2) and (6.3),

$$
\left\{\left(\zeta^{1-r^{2}}-1\right)+\left(\zeta^{2-r-r^{2}}-\zeta^{2-2 r}\right) \rho\right\} D=0 .
$$

From (6.4) and (6.5),

$$
\zeta^{r}\left(1-\zeta^{1-r}\right)\left(1-\zeta^{2 r+1}\right) \rho D=\left\{\left(\zeta^{1-r^{2}}-\zeta^{1-r}\right)-\left(\zeta^{2-r-r^{2}}-\zeta^{2-2 r}\right)\right\} \rho D=0 .
$$

Hence, $\pi^{2}(\rho D)=0$ and $\rho\left(\left(\zeta^{2}-1\right) /(\zeta-1)\right)^{2} \pi^{2} D=0$. Since $\rho$ and $\left(\zeta^{2}-1\right) /(\zeta-1)$ are in $\operatorname{Aut}(A)$, we have $\pi^{2}(D)=0$.

TheOREM 6.2. Let $N=\bigcap_{j=0}^{2} A\left[\zeta^{j}\left(1+\rho+\rho^{2}\right) \zeta^{-j}\right]$. Then we have $\operatorname{Ker}(\varphi)=N=A[\pi]$. 
Proof. Under the canonical projection $\varphi_{0}: F_{A} \rightarrow X_{0}=F_{A} /\langle\rho\rangle, \infty_{1}$ and $\infty_{2}$ are mapped onto the same point. Thus, $\operatorname{Ker}\left(\varphi_{0}\right)_{*}$ contains $A[\pi]$. Likewise, $A[\pi]$ is contained in $\operatorname{Ker}\left(\varphi_{j}\right)_{*}$. Thus

$$
A[\pi] \subseteq \operatorname{Ker}(\varphi) \subseteq N \subseteq A\left[\pi^{2}\right] .
$$

Let $D \in N$. Applying the endomorphism $w=\left(1+r \rho+r^{2} \rho^{2}\right) 1 / \pi^{2}$ to $\pi^{2} D=0$, we get

$$
\left(1+r \rho+r^{2} \rho^{2}\right) D=0 .
$$

Since $\left(1+\rho+\rho^{2}\right) D=0$ also, we obtain $\left\{(r-1) \rho+\left(r^{2}-1\right) \rho^{2}\right\} D=0$ or $(r-1) \rho\{1+(r+1) \rho\} D=0$. Since $D$ is a $p$-division point, $(p, r-1)=1$ and $\rho \in \operatorname{Aut}(A)$, it follows that $\{1+(r+1) \rho\} D=0$ or $(r-\rho) D=r\{1+$ $(r+1) \rho\} D=0$. Hence,

$$
A[\pi] \subseteq \operatorname{Ker}(\varphi) \subseteq N \subseteq A\left[\pi^{2}\right] \cap A[\rho-r] .
$$

By Lemmas 5.1 and 5.2, there is a $Q \in A\left[\pi^{2}\right]-A[\pi]$ such that $\rho(Q)=Q$ and $\rho(\pi Q)=r(\pi Q)$. Let $D=(a+b \pi) Q \in A[\rho-r]$, with $a, b \in \mathbf{F}_{p}$. Then $(a+b \pi) Q=(a r+b r \pi) Q$, whence $a=a r$ and $a=0$. Thus $D \in A[\pi]$ and $A\left[\pi^{2}\right] \cap A[\rho-r]=A[\pi]$. Hence, $\operatorname{Ker}(\varphi)=N=A[\pi]$.

Corollary 6.3. The isogeny $\varphi: A \rightarrow \prod_{j=0}^{2} \operatorname{Jac}\left(X_{j}\right)$ factors as

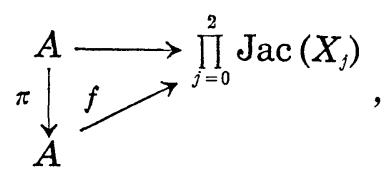

where $f: A \rightarrow \prod_{j=0}^{2} \operatorname{Jax}\left(X_{j}\right)$ is an isomorphism of abelian varieties defined over $K$.

Proof. We define an isomorphism $f: A \rightarrow \prod_{j=0}^{2} \operatorname{Jac}\left(X_{j}\right)$ of abelian varieties as follows. Given $D \in \operatorname{Pic}^{\circ}\left(F_{A}\right)$, let $E$ be such that $\pi E=D . \quad E$ exists since $\pi$ is an isogeny. Then we define $f(D)=\varphi(E) . f$ is well-defined and injective by definition. In particular, $f$ is a birational isomorphism of abelian varieties and hence an isomorphism of abelian varieties.

Let $C$ be the Klein quartic curve over $\mathbf{C}$ with projective equation

$$
X^{3} Y+Y^{3} Z+Z^{3} X=0 .
$$

$C$ has genus $3, \operatorname{Aut}(C) \approx P S L\left(2, \mathbf{F}_{7}\right)$, and the morphism 


$$
F_{1,2,4}^{7} \longrightarrow C, \quad(x, y) \longrightarrow\left((x-1) / y^{2}, \quad-(x-1) / y^{3}\right)
$$

is a birational isomorphism. Let $\operatorname{Jac}(C)$ be the Jacobian of $C$. We will denote by $\sigma$ and $\rho$ the following automorphisms of $C$ :

$$
\sigma:(x, y) \longrightarrow\left(\zeta^{4} x, \zeta^{5} y\right), \quad \rho:(x, y) \longrightarrow(1 / y, x / y),
$$

where $\zeta$ is a primitive 7 -th root of unity. Then by Proposition 2.1, we have the eximorphism

$$
\mathbf{Q}[\sigma, \rho] \longrightarrow \operatorname{End}^{0}(\operatorname{Jac}(C)) .
$$

By Theorem 4.1 and Corollary 6.3, we have

Corollary 6.4. Let $W=7^{-1}\left(1+r \rho+r^{2} \rho^{2}\right)(\sigma-1)^{4} \in \mathbf{Q}[\sigma, \rho]$, with $r=2$. Then End $(\operatorname{Jac}(C))=\operatorname{Im}(\mathbf{Z}[\sigma, \rho, W])$ and $\operatorname{Jac}(C)$ is isomorphic to a cube of an elliptic curve $E$.

Remarks. (1) From the Weierstrass equation for $E$ computed in [10], we see that $E$ is $J_{0}(49)$.

(2) As an application of Theorem 4.1, we give a second proof of the following result due to Prapavessi [10]: Let $\infty_{1}=(1,0,0), \mu_{j}=\zeta^{j}+\zeta^{-j}$ $(j \geq 0)$ and let $P=\left(\mu_{1}, \mu_{3}^{-1}, 1\right)$. Then $D=P+\rho P-2 \infty_{1}$ generates the kernel of $\pi^{3}$ over $\mathbf{Z}[\zeta]$. Prapavessi showed ([10], Lemma 2.1) that $\pi^{3}(D)=0$. It remains to show that $\pi^{2}(D) \neq 0$. Let $\infty_{2}=(0,1,0)$ and $\infty_{3}=(0,0,1)$. Suppose, on the contrary, that $\pi^{2}(D)=0$. Applying the endomorphism $\left(1-r^{2} \rho\right) 1 / \pi$ of $\operatorname{Jac}(C)$ we obtain $\left(1-r^{2} \rho\right) \pi D=0$, or

$$
\pi D=r^{2}\left\{\frac{\zeta^{r}-1}{\pi}\right\} \pi \rho D=r^{2}\left\{r+\frac{r(r-1)}{2} \pi+\cdots\right\} \pi \rho D=\pi \rho D .
$$

Since the group of $\pi$-division points on $\operatorname{Jac}(C)$ is generated by $\infty_{i}-\infty_{j}$ $(i \neq j), \pi\left(P-\rho^{2} P\right)=0$ follows from $\pi(D-\rho D)=0$. Hence there is a non-constant rational function $g$ on $C$ whose divisor is $\pi\left(P-\rho^{2} P\right)$. In particular, $g: C \rightarrow \mathbf{P}^{1}$ is a double covering, and $C$ is a hyperelliptic curve, which is a contradiction. This completes the proof that $\pi^{2}(D) \neq 0$.

(3) Our knowledge of the endomorphism ring of $A$ allows us to deduce a result of Greenberg [5] for $A=J_{1, r,-(1+r)}^{p}$. We have noted that $w=\left(1+r \rho+r^{2} \rho^{2}\right) 1 / \pi^{2}$ is an endomorphism of $A$ which is defined over $K$. Thus if $D \in A(K)$, then it follows that $w(D) \in A(K)$. Let $Q \in A\left[\pi^{3}\right]-A\left[\pi^{2}\right]$ be such that $\rho(Q)=r^{2} Q$. Setting $P=\pi^{2} Q$, we have $w(P)=(1+r \rho+$ $\left.r^{2} \rho^{2}\right)(Q)=3 Q$ is an element of $A(K)$. Let $\lambda, \mu \in \mathbf{Z}$ be such that $3 \mu+p \lambda$ 
$=1$. Then $Q=3 \mu Q \in A(K)$. Since $A\left[\pi^{3}\right]$ is a cyclic $\mathbf{Z}[\zeta]$-module with $Q$ as a generator, it follows that $A\left[\pi^{3}\right] \subseteq A(K)$. We also remark that the $p$-part of $A(K)$ is of the form $A\left[\pi^{3 l}\right]$ for some $l \geq 1$.

\section{Acknowledgements}

The author would like to thank Professor R. Coleman for his encouragement and support during the course of this work.

\section{REFERENCE}

[1] N. Aoki, Simple Factors of the Jacobian of a Fermat Curve and the Picard number of a Product of Fermat Curves, to appear in Amer. J. Math.

[2] R. Coleman, Torsion points on Abelian étale coverings of $\mathbf{P}^{1}-\{0,1, \infty\}$, Trans. AMS, 311 (1989), 185-208.

[ 3 ] R. Coleman, Lecture notes on Cyclotomy, Tokyo University (1985).

[4] R. Coleman and W. McCallum, Stable reduction of Fermat curves and Jacobi sum Hecke characters, J. Reine Angew. Math., 385 (1988), 41-101.

[ 5 ] R. Greenberg, On the Jacobian variety of some algebraic curves, Compositio Math., 42 (1981), 345-359.

[6] B. Gross and D. Rohrlich, Some results on the Mordell-Weil group of the Jacobian of the Fermat curve, Invent. Math., 44 (1978), 201-224.

[ 7 ] Y. Ihara, Profinite braid groups, Galois representations and complex multiplications, Ann. Math., 123 (1986), 43-106.

[ 8 ] N. Koblitz and D. Rohrlich, Simple Factors in the Jacobian of a Fermat curve, Canadian J. Math., 20 (1978), 1183-1205.

[9] C. H. Lim, Endomorphisms of Jacobian varieties of Fermat curves, to appear in Compositio Math.

[10] D. T. Prapavessi, On Jacobi sum Hecke Characters of Conductor a Power of 2. $\mathrm{Ph} . \mathrm{D}$. thesis, University of California, Berkeley (1988).

[11] D. Rohrlich, Appendix to "On the Periods of Abelian Integrals and a Formula of Chowla and Selberg" by B. Gross, Invent. Math., 45 (1978), 193-211.

[12] C. G. Schmidt, Der Definitions-Köper fur den Zerfall einer Abelschen Varietat mit Komplexer Multiplikation, Math. Ann., 254 (1980), 201-210.

[13] G. Shimura and Y. Taniyama, Complex multiplication of Abelian Varieties and its Applications to Number Theory, Tokyo, Math. Soc. Japan (1961).

Department of Mathematics

University of California

Berkeley

CA 94720, U.S.A.

Current address:

Department of Mathematics

Faculty of Science

National University of Singapore

Kent Ridge

Singapore 0511 